\title{
Der Stand des Zwerchfelles bei Gesunden und Emphysematikern.
}

\author{
Von \\ Dr. med. Seefeldt.
}

Ich habe es mir in Folgendem zur Aufgabe gestellt, die Grösse der Zwerchfellexkursionen sowie die exspiratorische und inspiratorische Ruhesteliung des Diaphragmas bei ruhiger und bei willkürlich vertiefter Atmung im Röntgenlicht zu verfolgen und orthodiagraphisch festzulegen.

Die Tersuche sind angestellt sowohl an Leuten mit gesunden Lungen wie auch an zahlreichen Emphysematikern.

Zu grösstem Dank verpflichtet bin ich Herrn Prof. Dr. B r a u e r, welcher mir das Material seiner Klinik höchst liebenswürdiger Weise zur Verfügung stellte und dem Oberarzt der Klinik, Herrn Priratdozent Dr. Bruns, welcher mir bei der Aufstellung des Untersuchungsplanes zur Seite stand und die Röntgenuntersuchungen rornahm.

Bevor ich nun zur eigenen Arbeit übergehe, und zur Erläuterung der angeregten Fragen, muss ich die Angaben zusammenstellen, die Hofbauer und Holzknecht in ihrer Arbeit, zur Physiologie und Pathologie der Atmung“" gemacht haben. Gelegentlich seiner pneumographischen Versuche fand $\mathrm{H}$ ofbauer an den aufgezeichneten Atemkurven eine „eigentümliche immer wiederkehrende Erscheinung“. Diejenigen Atem-Zacken der Kurven nämlich, welche grösser als die übrigen waren, überragten fast nie nach beiden Seiten hin gleichmässig die anderen. Lediglich nach der inspiratorischen Seite hin machte sich der Zuwachs bemerkbar. Diese Beobachtung führte ihn zu der Frage, 
ob die Exkursionen des Zwerchfells bei Vertiefung der Atmung in demselben Sinne sich ändern oder nicht, ob auch hier nur eine Vermehrung der inspiratorischen Entfernung der Brustwand vom Thoraxzentrum sich feststellen lasse, oder ob In- und Exspiration gleichmässig an der Vertiefung teilnehmen. Zur Lösung dieser Frage stellten dam Hofbauer und Holzknecht neue Untersuchungen unter Zuhilfenahme des Röntgenverfahrens an. Diese uns interessierenden Versuche sind in der neunten und elften Mitteilung des Bandes: "Zur Physiologie und Pathologie der Atmung": Mitteilungen aus dem Laboratorium für radiologische Diagnostik und Therapie im $k$. $k$. allgemeinen Krankenhause in Wien 1907 reröftentlicht. Wir lesen dort auf Seite 54:

„Es kommt nur überaus selten vor, dass bei vertiefter Atmung die Inspiration um ebensoviel von der der ruhigen Atmung zukommenden Inspirationsstellung abweicht, wie die Exspiration von der der ruhigen Atmung zukommenden Exspirationsstellung. Gewölnnlich ist keine so gleichmässige Atmung zu bemerken. Vielmehr macht sich in beinahe allen Fällen die Vertiefung der Atmung in der Form geltend, dass lediglich die Inspiration vertieft wird, so dass also sich bei der Inspiration eine wesentliche und weitgehende Verstärkung der Atmung einstellt, während eine solche bei der Exspiration eigentlich fehlt. Infolgedessen bleibt dann am Ende der Exspiration das \%werchfell bei rertiefter Atmung auf derselben Höhe stehen, welche es schon bei rubiger unbeeinflusster Atmung innegehabt hatte. Nur bei der vertieften Inspiration entfernt sich das Zwerchfell von seiner inspiratorischen Ruhestellung.

Es vollzieht sich bei der Mehrzahl der Menschen die Vertiefung der Atmung lediglich dadurch, dass die Einatmung eine verstärkte wird, während die Ausatmung nichts an Stärke gewinnt. In vielen Fällen geht die Verschiebung der in- und exspiratorischen Wirkungssphäre so weit, dass die Inspirationsbewegung eine stark gesteigerte ist, die Exspiration nicht nur nicht gesteigert, sondern sogar in ihrer Intensität gegenüber der Torm herabgesetzt ist. In solchen Fällen beschränkt sich aber immerhin die Verringerung der Exspirationsintensität darauf, dass das Zwerchfell bei vertiefter Ausatmung sich dem Zentrum des Thorax weniger nähert als bei ruhiger Exspiration, es tritt aber doch während der vertieften Exspiration höher kranial als bei der ruhigen Inspiration. Bei andern Fällen hingegen kommt es zu einer so wesentlichen Verringerung der exspiratorischen Leistung, dass das Zwerchfell am Ende der Ausatmung bei forcierter Respiration weniger hoch in den Thorax hineintritt als während der Einatmung bei ruhiger Respiration. Bei diesen Fällen also bleibt die Lunge selbst 
nach der Ausatmung noch viel mehr gebläht als dies bei ruhiger Atmung selbst am Ende der Einatmung, also bei grösstmöglichster Entfaltung der Lunge der Fall war. Dieser unzweckmässige Mechanismus liesse es nun begreiflich erscheinen, wenn infolge einer solchen geänderten Atemmechanik sich konsekutiv Lungenblähung einstellte. Es wird uns dadurch geradezu Einblick gewährt in die pathologische Physiologie des Emphysems, in den Mechanismus, welcher statthat, wenn bei Behinderung der Atmung sich konsekutiv Lungenblähung einstellt.

Ein Einwand könnte gegen die Richtigkeit dieser Suppositionen erhoben werden. Als Grundlage für die eben dargelegten Schlussfolgerungen wurden lediglich die Resultate der Beobachtung der $\%$ werchfellexkursionen bei ruhiger und vertiefter Atmung herangezogen, und wir supponierten, ohne dieses erst zu beweisen, dass auch die Bewegungen der Thoraxwand sich in genau derselben Weise bei Vertiefung der Atmung verhalten wie die des Diagramms. Nun liegt der Einwand nahe, dass vielleicht $Z$ werchfell und Thoraxwand sich verschieden verhalten, dass mit anderen Worten es nicht angehe, ohne weiteres anzunehmen, dass die Vertiefung der Atmung im gleichen Sinne von seiten der Rippen und des Zwerchfells statt habe. Man könnte denken, dass etwa bloss das Zwerchfell eine solche Präponderanz der inspiratorischen Bewegungen aufweise, während den Rippen eine entsprechende Verstärkung der exspiratorischen Leistung bei der Vertiefung der Atmung zufalle. Bei näherem Zusehen ergeben sich schon rein theoretische Schwierigkeiten, welche sich bei einer solchen Rollenverteilung im Atemspiel einstellen müssten. Zur Gewissheit wird die Hinfälligkeit dieser Hypothese von einem verschiedenen Respirationsspiel von Zwerchfell und Thoraxmuskulatur mit Rücksicht auf die Versuche von $Z$ wardemaaker (Archiv für Anatomie und Physiologie, physiologische Abteilung 1904). Dieser Autor konnte schon vor längerer Zeit nachweisen, dass beim Menschen ein vollständiger und unabänderlicher Synchronismus der Atembewegungen des Zwerchfells einerseits und des knöchernen Thorax andererseits besteht. Dadurch wird die Gewähr dafür gegeben, dass die Berechtigung vorliegt, aus der Beobachtung der Zwerchfellbewegungen allein Schlüsse zu ziehen für den Mechanismus der Gesamtatmung (sowohl des kostalen als des diaphragmalen Anteils der Atembewegungen)." Ausserdem ergaben ja auch $\mathrm{H}$ ofbauers pneumographische Kurven, dass bei Vertiefung der Atmung nur die inspiratorischen Brustwandexkursionen an Grösse zunehmen.

Im Resïmee stellen dann beide Autoren folgende Sätze auf: „Die Vertiefung der Atmung wird von der 
Inspiration bestritten. Ursache: Die auf elastischen Kräften beruhende Exspiration ist einer Steigerung nicht fähig, wohl aber die muskuläre Inspiration. Die Verwendungderexspiratorischen Auxiliarmuskeln bleibt zunächst aus, weil diese die Heranziehung eines fremden ungebahnten Mechanismus bedeuten wïrde.

Bei tiefer Atmung entwickelt sich also auch bei ganz physiologischen Verhältnissen meist eine Überdehnung der Lunge, indem auf die verstärkte inspiratorische Blähung gar keine Rïckkehr in einen entsprechend weniger gedehnten Zustand während der Ausatmung folgt. Diese physiologische Erfahrung vermaguns ohne weitereszu erklären, warum es bei gesteigertem Atembedürnis und Lufthungerzur Ausbildung des Volumen pulmonum auctum und weiterhin zur Ausbildung des Emphysems kommen muss. Die Lunge wird bei der vertieften Atmung stetig überdehnt, und niemals wird ihr Gelegenheit geboten, sich entsprechend zusammenzuziehen. Es resultiert also ein Blähungszustand der Lunge infolge vertiefter Atmung trotz normaler anatomischer Verhältnisse."

Bei unseren Versuchen, die wir mit dem Grödelschen Orthodiagraphen zuerst an Gesunden anstellten, fanden wir recht erhebliche Schwierigkeiten, objektive und untereinander vergleichbare Werte zu eruieren.

Sie wurden hervorgerufen erstens durch die entgegengesetzten Bewegungen, welche Brustwand und Zwerchfell während der einzelnen Atmungsphasen ausführen, und dann durch die inkonstante Körperhaltung der Cntersuchten, indem dieselben während der Periode der (ihnen aufgetragenen) vertieften Atmung den Brustkorb unwillkürlich wölben, d. h. sie gehen durch Extension der Brustwirbelsäule ron selbst in eine mehr aufrechte Haltung über. Andererseits sinken besonders die alten, hinfälligen Emphysematiker, ohne es zu wollen, gelegentlich während der Dauer der Durchleuchtung etwas vorn über oder in sich zusammen. Dadurch werden stets andere Zwerchfellteile schattenrandständig und das Lungenvolumen ändert sich auch bei völliger Körperruhe.

Die Zunahme der Zwerchfellexkursionen beim Übergang aus der ruhigen in die tiefe Atmung erkennt man nicht aus dem Abstand der in- und exspiratorischen Zwerchfellschattengrenzen; denn erstens werden bei der während der vertieften Inspiration eintretenden Thoraxhebung gleichzeitig die vorderen Zwerchfellansätze mit in die Höhe 
genommen. Andererseits werden bei der exspiratorischen Thoraxsenkung ebenfalls die Zwerchfellansätze mit hinabgenommen. Es kreuzen sich also Zwerchfell- und Brustwandbewegung bei forcierter Respiration.

Die wahre Grösse der Zwerchfellexkursionen bei vertiefter Atmung ist also nur zu erkennen durch den Vergleich des Zwerchfellstandes mit dem der bleiernen Rippenmarken.

Als Kontrolle und Ergänzung der orthodiagraphischen Untersuchung haben wir noch das Bandmass verwandt, das uns über das Vorhandensein oder Nichtvorhandensein einer Erweiterung des Thorax bezw. der Lunge bei den einzelnen Fällen ziemlich sicheren Aufschluss ausserdem geben konnte. Diese Bandmassbestimmungen wurden sehr exakt ansgeführt; durch Hunderte von Messungen hierzu und zu anderen Versuchen wurde die Sicherheit erlangt, die zu einem einigermassen sicheren Schluss auf Grund dieser Messbestimmungen berechtigen darf.

In Erwägung dieser Punlite und auf Grund zahireicher Vorversuche kamen wir zu folgender Technik:

Diagraphiert wurde stets bei sagittaler Strahlenrichtung gelegentlich zur Kontrolle auch in frontaler Richtung. Bei jedem Versuch wurde die Thoraxumrandung vor, während und nach der vertieften Atmung diagraphiert zwecks besserer Schätzung des Lungenvolumens.

Die 5. und 6. Rippen trugen rechts wie links Kontrollbleimarken. Die Zwerchfellschattengrenze wurde aufgezeichnet in inspiratorischer wie in exspiratorischer Ruhestellung ror, während und unmittelbar nach der vertieften Atmung.

Die Lage der Marken wird bei In- und Exspirationsstellung des Thorax auf dem Diagramm festgelegt. Nach Beendigung des Versuchs werden die bei Exspirationsstellung des Thorax gezeichneten Kontrollmarken durch eine Linie miteinander verbunden.

Wir befahlen unseren Versuchspersonen (meist Jägern des hiesigen Bataillons) nach einer Periode ruhiger Atmung tief zu atmen, ohne irgendwelche genaueren Instruktionen über die Art und Tiefe der Ausatmung.

Die Resultate unserer Untersuchungen sind folgende:

Während der vertieften Atmung, d. h. nachdem schon 40-50 vertiefte Atemzüge vorhergegangen sind, steht das $Z$ werchfell am Schluss der Exspirationsphase tiefer, d. h. ferner vom Thoraxzentrum, als bei ruhiger gewöhnlicher Atmung. Die Stellung des Zwerchfells am Ende der Inspiration bei rertiefter Atmung ist sehr variabel; meist steht das Zwerchfell tiefer als ror der Periode der tiefen Atemzüge, und zwar auch in den Fällen, bei denen durch die Atem- 
vertiefung die Haltung aufrechter und durch das Emporheben des Brustkorbes die vorderen Zwerchfellinsertionen mit in die Höhe genommen waren. Xach Übergang aus der vertieften in die ruhige Atmung. steht das Zwerchfell am Ende der Exspiration in den meisten Fällen $t$ i e f e $\mathrm{r}$ als vorher bei der ruhigen gewöhnlichen Atmung. Dieser Tiefstand des Zwerchfells nach Abschluss der Periode der vertieften Atmung weist auf ein Volumen pulmonum auctum hin und entspricht den Resultaten $\mathrm{Hofb}$ a u rs und Holzknechts.

In der untenstehenden Tabelle über Gesunde ist gerade so wie bei der Tabelle über Emphysematiker der Abstand der Zwerchfellschattengrenze von einer die in Exspirationsstellung gezeichneten Rippenmarken rerbindenden Horizontallinie in Zentimetern angegeben. Der Abstand wurde mit plus bezeichnet, wenn die diagraphierte Zwerchfellinie sich unter der Rippenmarkengrenze befand, mit minus, wenn der höchste Punkt der Zwerchfelkuppe diese Grenze nach oben überragte, mit anderen Worten, wenn das Zwerchfell sich dem Thoraxzentrum genähert batte.

\section{Tabelle über gesunde Personen.}

P. 1. Bei ruhiger Atmung rechts, minus $0,8 \mathrm{~cm}$ liuks, plus $0,5 \mathrm{~cm}$

B. 1. Bei ruhiger Atmung rechts, plus $0,9 \mathrm{~cm}$ links, , $1, \check{\partial}$,

G. 1. Bei rubiger Atmung rechts, minus $2,8 \mathrm{~cm}$ links, " 3,0 ,

B. 1. Bei ruhiger Atmung rechts, gleich links, plus $0,7 \mathrm{~cm}$
2. Nach 40 vertieften Atemzägen rechts, plus $1,0 \mathrm{~cm}$ links, , 1,6 ,

2. Nach 40 vertieften Atemzügen. rechts, plus $3,0 \mathrm{~cm}$ links, , 2,5,

2. Nach 50 vertieften Atemzügen rechts, minus $1,3 \mathrm{~cm}$ links, " 1,0 ,

2. Nach 40 vertieften Atemzügen rechts, minus $0,4 \mathrm{~cm}$ links, gleich.

Der Befund, dass das Zwerchfell nach Aufhören der vertieften Atmung bei Exspiration tiefer steht, als vor Beginn der vertieften Atmung, lässt schliessen auf eine Vergrösserung des Thoraxinnern und damit des Lungenvolumens. Dass es sich dabei um keine wesentliche Vergrösserung des Thoraxinnern handeln kann, geht aus meinen kyrtometrischen Untersuchungen hervor, die ergaben, dass der Brustumfang, den ich in der Mamillarlinie nach der Periode der tiefen Atemzüge feststellte, sich kaum geändert hat.

Brustkorb und Lungen brauchen bei stärkerer inspiratorischer Dehnung eine entsprechend längere Zeit, um sich zum normal-exspira- 
torischen Volumen verkleinern zu können. Die steht ihnen aber bei derartigen Versuchen meist nicht zur Verfügung, indem die Atemfrequenz im Vergleich zu der bei der Ruheatmung eher gesteigert wird ( $\mathrm{T}$ endeloo, Hofbauer). Bei der neueinsetzenden vertieften Inspirationsbewegung ist also der Thorax noch etwas erweitert. Ebenso sind die Alveolen speziell die mehr peripher gelegenen, dehnbareren und stärker gedehnten Alveolen noch eine Zeitlang erweitert. Die elastische Nachwirkung an Brustkorb wie Alveolen wird erst nach Aufhören der vertieften Atmung aus der unvollkommenen eine vollkommene.

So demonstriert also die Tatsache, dass das Zwerchfell noch einige Zeit nach Beendigung der Serie der tiefen Atemzüge in seinem Tiefstand verharrt, dass der Brustkorb den durch die zahlreichen und starken Dehnungen bewirkten Spannungszustand einige Zeitlang beibehält.

Liegen normale anatomische Verhältnisse vor, sind insbesondere die oberen und tieferen Luftwege von normaler Weite, so spielt für die hier besprochene temporäre Brustkorberweiterung die Form und Dauer der auf elastischen Kräften beruhenden Fxspiration keine Rolle, denn bei normalen Luftwegen gehen Brustwand- und Lungenverkleinerung absolut parallel. Für die Dauer der exspiratorischen Verkleinerung der Alveole kommt die Menge der in ihr enthaltenen Iult nicht in Betracht, sondern nur der Grad der inspiratorischen Dehnung.

Ein H in der $n$ is für die Retraktion gedehnten elastischen Lungengewebes bildet die intraalveolare Luft nur dann, wenn die Bronchiolen durch katarrhalische Schwellung oder Bronchialmuskelkrampf primär verengt, durch die exspiratorische Erhöhung des intrapulmonalen Druckes noch weiterhin stenosiert bezw. rerschlossen werden.

Einer aktiven Dehnung der Lungen in der Exspirationsphase begegnen wir nur bei willkürlicher oder pathologischer Stenose der oberen Luftwege oder deren Öffnungen nach aussen und gleichzeitiger aktiv muskulärer Exspiration.

Dann werden die unteren Lungenabschnitte durch das emporgetriebene Zwerchfell komprimiert. Die aus diesen Partien vertriebene Luft kann wegen der Verengerung der oberen Luftwege nicht rasch genug nach aussen entweichen und "staut" sich daher in den oberen Lingenabschnitten, diese aktir dehnend und aufblähend (Hustenemphysem).

Ije willkürliche Vertiefung der Atmung und die dadurch erzielte, temporäre Füllungszunahme der Lunge stellt eine Lungengymnastik 
dar. Die Erweiterung, aber dabei zugleich Zunahme der Leistungsfähigkeit der Lunge des Sportsmanns entsteht auf Grund einer entsprechend häufig wiederholten Lungengymnastik.

So nahm nach Untersuchungen, die im Kopenhagener physiologischen Institut durch $\mathrm{Hasselbalch}$ vorgenommen wurden, das mittlere Lungenrolumen eines Mannes, der täglich 1/2 Jahr lang seine Lungenkraft am Spirometer übte, um $500 \mathrm{ccm}$ zu.

Die Cintersuchungen über den Stand des Zwerchfells bei Atemvertiefung haben wir dann auch auf Em physematiker mit starrem Thorax ausgedehnt. Das Resultat bei mechano-therapeutisch noch nicht beeinflussten Fällen war, dass wir nur bei einem Teil der Untersuchten einen Tiefstand des Zwerchfells nach Abschluss der Periode der vertieften Atmung feststellen konnten.

\section{Tabelle über Emphysematiker.}

Patient M. 1. Bei ruhiger Atmung.

Rechts, gleich.

Links, minus $1,0 \mathrm{~cm}$

Patient G. 1. Bei ruhiger Atmung. Rechts, gleich.

Links, plus $1,9 \mathrm{~cm}$

Patient Z. 1. Bei ruhiger Atmung. Rechts, plus $2,7 \mathrm{~cm}$ Links, plus $3,2 \mathrm{~cm}$

Patient B. 1. Bei ruhiger Atmung. Rechts, plus $6,2 \mathrm{~cm}$ Links, plus $6,9 \mathrm{~cm}$
2. Nach 40 vertieften Atemzügen. Rechts, plus $0,2 \mathrm{~cm}$ Links, plus $0,3 \mathrm{~cm}$

2. Nach 50 vertieften Atemzïgen. Rechts, plus $1,3 \mathrm{~cm}$ Links, plus $2,9 \mathrm{~cm}$

2. Nach 40 vertieften Atemzügen. Rechts, plus $2,0 \mathrm{~cm}$ Links, plus $3,6 \mathrm{~cm}$

2. Nach 40 vertieften Atemzügen. Rechts, plus $7,5 \mathrm{~cm}$ Links, plus $7,3 \mathrm{~cm}$

Kranke mit starkem Emphysem bekamen durch die Vertiefung der Atmung keine weitere Zunahme des Dehnungszustandes ihrer I.ungen.

Freund kommt auf Grund seiner vor 50 Jahren begonnenen und später fortgeführten Untersuchungen zu der Überzeugung, dass ein primär starr dilatierter Thorax die Ursache eines alveolären Emphysems sein könne. Nach Freund können ungefähr vom 16. Lebensjahr bis ins hohe Alter hinauf die Rippenknorpel durch Ernährungsstörungen degenerieren; sie nehmen eine schmutzig gelbe Farbe an, zerfasern, werden dann später durch Einlagerung von Kalksalzen weniger elastisch, schliesslich starr. Während bei manchen Fällen 
die Anfänge der Erkrankung sich nur zuerst an dem zweiten und dritten Rippenknorpel zeigen, um von hier aus dann fortzuschreiten, werden bei anderen Fällen gleichzeitig alle Rippenknorpel ergriffen; diese Rippenknorpelanomalie ruft nun nach Freund an der Gestalt und Funktion des Thorax insofern Veränderungen hervor, als der durch die Krankheit veränderte voluminösere Rippenknorpel die Rippen auseinanderdrängt und sie in eine entgegen ihrer sonstigen Lage veränderte inspiratorische Stellung bringt. Gleichzeitig wird das Sternum je nach dem Sitze der Erkrankung mit nach oben oder seitlich gehoben. Wenn diese Bewegung des Brustbeins und der Rippen an die durch die mechanische Einrichtung bestimmte Grenze gelangt ist, und sich dann der Knorpel noch weiter vergiössert, so wird in dem betroffenen Rippenring und später im ganzen Thoraxgebäude ein dauernder Spannungszustand hervorgerufen; der Rippenknorpel muss sich schliesslich nach aussen über einen kürzeren Radius beugen. Bei einseitigenn Ergriffensein trete die Asymmetrie des Thorax als Folgeerscheinung zutage, die sogenannte partiell fortschreitende starre Dilatation des Brustkastens; bei gleichzeitigem und gleichmässigem Erkranktsein sämtlicher Rippenknorpel wäre die allgemeine starre Dilatation des Thorax der Ausgang. Aus allen diesen Veränderungen zusammen resultiere eine dauernde Erweiterung des Brustkorbes in Inspirationsstellung, die durch Zerren an der ihm adhärenten Lunge die primäre Ursache eines alveolären Emphysems sein könne.

Auch die Frage der Thoraxstarre haben wir an unserem Material untersucht.

Nachstehende Tabelle gibt eine Übersicht iiber die maximale Dehnungsfähigkeit des Thorax bei unseren Emphysematikern.

\section{Brustumfänge in Mamillarlinie bei tiefster Inspiration und Exspiration.}

$\begin{array}{lrll}\text { Fall 1. } & 96,8: 100,0 \mathrm{~cm} & \text { Fall } 7 . & 86,0: 91,0 \mathrm{~cm} \\ \text { Fall 2. } & 90,5: 91,3 \mathrm{~cm} & \text { Fall } 8 . & 82,0: 84,0 \mathrm{~cm} \\ \text { Fall 3. } & 107,8: 109,0 \mathrm{~cm} & \text { Fall } 9 . & 81,0: 83,5 \mathrm{~cm} \\ \text { Fall 4. } & 93,5: 96,5 \mathrm{~cm} & \text { Fall } 10 . & 88,0: 89,2 \mathrm{~cm} \\ \text { Fall 5. } & 80,5: 85,0 \mathrm{~cm} & \text { Fall } 11 . & 76,9: 80,4 \mathrm{~cm} \\ \text { Fall 6. } & 89,0: 91,2 \mathrm{~cm} & \text { Fall } 12 . & 75,8: 78,2 \mathrm{~cm}\end{array}$

Es geht aus dieser Tabelle hervor, dass so gut wie alle Emphysematiker einen starren oder nahezu starren Thorax haben. Die Atmung geschieht bei ihnen hauptsächlich durch Hebung des Thorax mit den an die Wirbelsäule und Schädel ansetzenden Inspirationsmuskeln. Ferner wirken mit die völlig normal gebliebenen Atembewegungen des Zwerchfells. Auch diese finden wir in nachfolgender 
Tabelle zusammengestellt und mit den Zwerchfellexkursionen von fünf gesunden jugendlichen Menschen verglichen.

\begin{tabular}{|c|c|c|c|c|c|}
\hline \multirow{2}{*}{ Emphysematiker } & \multicolumn{3}{|c|}{ Bei gewöhnlicher Atmung } & \multicolumn{2}{|c|}{ Bei vertieften Atemzügen } \\
\hline & rechts & $\vdots$ & links & rechts & links \\
\hline $\mathrm{N}$. & 1,3 & ; & 1,5 & 1,8 & $3, \tilde{5}$ \\
\hline G. & 0,5 & & 2,0 & 2,3 & 2,0 \\
\hline $\mathrm{Z}$. & 0,8 & & 0,8 & 0,3 & 0,3 \\
\hline G. & 1,0 & & 1,4 & 0,8 & 2,8 \\
\hline M. & 1,9 & & 1,4 & 2,8 & 2,0 \\
\hline B. & 0,9 & & 1,0 & 0.9 & 0,9 \\
\hline $\mathrm{E}$. & --- & &.- & $\cdots$ & \\
\hline G. & - & & - & $\cdots$ & \\
\hline \multicolumn{6}{|l|}{ Gesurde } \\
\hline G. & 1,5 & & 1,4 & 1,0 & 1,3 \\
\hline B. & 2,0 & & 3,0 & 1,6 & 2,1 \\
\hline F. & 1,4 & & 1,9 & 2,0 & 2,0 \\
\hline P. & 1,0 & & 0,8 & 0,4 & 0,8 \\
\hline Pf. & 1,0 & & 1,5 & 2,5 & 2,5 \\
\hline
\end{tabular}

Betrachten wir run unsere Fälle auf die ron Fre und gemachten Beobachtungen, so finden wir in der Tat auf den Röntgenbildern fast stets starkes Ergriffensein der Rippenknorpel durch den krankhaften Verknöcherungsprozess.

Aus den zum Schlusse beigefügten Krankengeschichten und Aufzeichnungen über die Emphysematiker und aus den dazu gehörigen Diagrammen fanden wir, dass gelegentlich nach Abschluss (bei den Fällen Nr. 1, 4, 5, 11), einer Körperarbeit das Zwerchfell bei Exspiration wesentlich höher treten könne als zuvor; die Erklärung finden wir vielleicht darin, dass durch die vermehrte Respiration während der Arbeit Hindernisse für den exspiratorischen Luftstrom, wie Bronchial-Schleim und Eiter, weggeschafft' werden können.

\section{Krankengeschichten.}

\section{Versuche und Befunde an Emplysematikern.}

1. H. M., 56 Jahre, Grösse $171 \mathrm{~cm}$. Emphysem.

Die Anamnese über Familie ergibt nichts Bemerkenswertes. Selbst als Find und in der Jugend gesund, mit 20 Jahren Müllerknecht in schwerem Dienst - Sackträger - diesen Anstrengungen wird die Schuld für die Erkrankung gegeben. Seit mehreren Jahren Husten und Atemnot, besonders beim Steigen, besondere Beschwerlichkeit beim Ausatmen. 
Befund: Kräftig gebauter Majn, kein Fettansatz; symmetrisch gebauter, mittelstarker, gewölbter Thorax, erscheint nach oben gezogen, kurzer Hals, beim Atmen angespannte Muskulatur, Elastizität des Thorax in den unteren Teilen gut erhalten. Die geräuschvolle Atmung beschleunigt. Eine Eigentümlichkeit der Atmung fällt hier auf. Aus der tiefsten Inspiration kann er nicht sofort anf Aufforderung ausatmen, der Brustkorb bleibt eine ganze Weile gebläht stehen, bis durch einen Hustenstoss ein kleiner 'leil Luft entleert werden kann. Dann bleibt der Thorax wieder stehen. Die weitere Entleerung der Lunge findet dann jedesmal in derselben Weise durch Expression einer kleinen Menge Luft durch Hustenstösse statt, bis die tiefste Exspirationsstellung erreicht wird. Die Kehl. kopfspiegelung ergibt chronische Laryngitis. Bauchmuskulatur stark. Die Lungengrenzen binten am unteren Rand 10. Rippe nach oben und unten deutlich verschieblich. Abdominaler Atemtypus, starke Bronchitis. Vitalkapazität nicht bestimmbar.

\begin{tabular}{cc}
\hline Neigungswinkel (Freund) & $\begin{array}{c}\text { sagittaler Durchmesser in der } \\
\text { Neigungsebene }\end{array}$ \\
\hline bei gewöhnlicher Atmung $30^{\circ}$ & $14 \mathrm{~cm}$ \\
bei tiefster Inspiration $26^{\circ}$ & $14^{1 /+} \mathrm{cm}$ \\
\hline
\end{tabular}

Sagittaler horizontaler Durchmesser bei gewöhnlicher Atmung

bei tiefster Inspiration

Oberer Sternalrand $16 \% \mathrm{~cm}$

Mitte Sternum $21 \% \mathrm{~cm}$

Processus ensiformis $21 \frac{1}{4} \mathrm{~cm}$
$16 \% \mathrm{~cm}$
$22 \% \mathrm{~cm}$
$21^{3}, \mathrm{~cm}$

$96^{3} / 4: 97^{1 / 4} \mathrm{~cm}$

$96^{3} / 4: 100 \mathrm{~cm}$

$87: 87^{1}: 2 \mathrm{~cm}$

$86^{1,4}: 88^{1} / 2 \mathrm{~cm}$.

Röntgenbefund: Bei der Durchleuchtung erweist sich das Zwerchfell recht gut beweglich, es macht ausgiebige Atembewegungen. Die eigentümliche Art der Ausatmung kann man deutlich auf dem Schirm erkennen. Das Zwerch. fell geht immer ruckweise nach oben. Auf der Röntgenplatte sieht man Rippenknorpel 1 und 2 manschettenförmig verknöchert.

J. M., 73 Jahre, Grösse $163 \mathrm{~cm}$. Emphysem.

Die Anamnese über die Familie bietet nichts Bemerkenswertes. In der Jugendzeit stets gesund gewesen, war Soldat. Seit 6-7 Jahren Asthma, Husten mit Atemnot, jede Anstrengung verursacht starke Atembeschwerden.

Befund: Mittelmässig kräftig gebaute Person, wenig Fettansatz. Ziemlich gleichmässig geformter 'Thorax, schwach fassförmig, fast völlig starr, ohne Elastizität. Der ganze Thorax erscheint nach oben geboben, Halsmuskulatur wird beim Atmen stark in Anspruch genommen. Atmung beschleunigt, erschwert, 
keuchendes Ein- und Ausatmen. Bauchmuskulatur gut. Die Langengrenzen hinten Mitte 10. Rippe, exspiratorisch und inspiratorisch leidlich verschieblich. Bronchitis. Rein abdominaler Atemtypus. Vitalkapazität $1600 \mathrm{ccm}$.

\begin{tabular}{cc} 
Neigungswinkel (Freund) & $\begin{array}{c}\text { Sagittaler Durchmesser in der } \\
\text { Neigungsebene }\end{array}$ \\
bei gewöhnlicher Atmung $29^{\circ}$ & $13 \mathrm{~cm}$ \\
bei tiefster Inspiration $28,5^{\circ}$ & $13 \mathrm{~cm}$ \\
\hline
\end{tabular}

Sagittaler horizontaler Durchmesser bei gewöhnlicher Atmung

Oberer Sternalrand $14 \mathrm{~cm}$

Mitte Sternum 20,5 cm

Processus ensiformis $21,5 \mathrm{~cm}$ bei tiefster Inspiration
$14,1 \mathrm{~cm}$
$20,6 \mathrm{~cm}$
$21,6 \mathrm{~cm}$

Unfang in Mamillarlinie bei gewöhnlicher Atmung: $\quad 90,5: 90,7 \mathrm{~cm}$ bei tiefster Exspiration und Inspiration: $90,5: 91,3 \mathrm{~cm}$ Umfang des Bauches in Nabelhöhe bei gewöhnlicher Atmung: 91,5:92,5 cm bei tiefster Exspiration und Inspiration: $89,0: 93,5 \mathrm{~cm}$.

Röntgenbefund: Das Zwerchfell erweist sich entsprechend dem perkutorischen Befund gut beweglich. Auf dem Röntgenbilde erscheinen die Rippenknorpel bis zum 5 . hinab verkalkt.

3. V. Sch., 57 Jahre, Grösse $170 \mathrm{~cm}$. Emphysem.

An amese: Vater an Altersschwäche, Nutter an Lungenschlag gestorben Sie hat einen sehr "hohen" Brustkorb gehabt und immer an Husten und Atembeschwerden gelitten. Zwei Brüder und eine Schwester sind an Asthma zugrunde gegangen, hatten dieselbe hohe Brustbeschaffenheit. Als Kind gesund, ebenso später. Normaler Brustumfang während der Militärzeit. Als Bergmann im Kupferbergwerk schwere Arbeit bei gebückter Haltang. Diesen Anstrengungen gibt er die Schuld für seine Erkrankung, die seit ungefähr 5 Jahren bestände. Sie habe mit Kurzatmigkeit und Husten angefangen beim Gehen, er habe gemerkt wie sein Brustkorb allmählich immer grössere Dimensionen angenommen habe.

Befund: Sehr starker Knochenbau, wenig Fettansatz, ein massig entwickelter, hoch gewölbter', stark nach oben gezogener Thorax, stark dilatiert. Die Elastizität fast aufgehoben, der ganze Brustkorb wird beim Atmen mit nach oben genommen, die Halsmuskulatur ist angestrengt, Atmung beschleunigt, von Stridor begleitet, Ausatmung beschwerlich. Bauchatmung. Bauchmuskulatur gut. Die Lungengrenzen stehen hinten am unteren Rand der 10. Rippe, sind ver. schieblich. Bronchitis. Vitalkapazität $1800 \mathrm{ccm}$.

Neigungswinkel (Freund)

bei gewöhnlicher Atmung 22,0

bei tiefster Inspiration 21,0
Sagittaler Durchmesser in der Neigungsebene
$14^{1 / 4} \mathrm{~cm}$

$14 \% 2 \mathrm{~cm}$ 


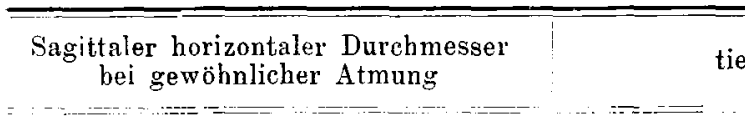

Oberer Sternalrand $18 \mathrm{~cm}$

Mitte Sternum $24 \mathrm{~cm}$

Processus ensiformis $27 \mathrm{~cm}$ tiefste Inspiration

$$
\begin{aligned}
& 18^{1}+\mathrm{cm} \\
& 24^{1}+\mathrm{cm} \\
& 27^{1 / 2} \mathrm{~cm}
\end{aligned}
$$

Cmfang in Namillarlinie bei gewöhnlicher Atmung:

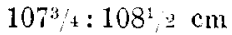

bei tiefster Exspiration und Inspiration: $107^{3 / 4}: 109 \mathrm{~cm}$

Cmfang des Bauches in Nabelhöhe bei gewöhnlicher Atmung: $111: 111^{1 / 4} \mathrm{~cm}$ bei tiefster Exspiration und Inspiration:

$110: 112 \mathrm{~cm}$.

Rïntgenbefund feblt.

4. H. G., 49 Jahre: Grösse $166 \mathrm{~cm}$. Emphysem.

Anamnese: Vater an Phthise gestorben, Nutter lebt, leidet an Atembeschwerden, Husten, dyspnoischen Zuständen (Asthma). An Geschwistern nichts Besonderes. In der Jugend gesund, wurde Hüttenarbeiter an einer Eisenhütte, glaubt durch das Heben der schweren Eisenplatten sein Leiden bekommen zu haben, das ungefähr 10 Jahre besteht. Seit 4 Jahren Zunahme der Atembeschwerden (Atemnot), seit dieser Zeit arbeitsunfähig.

Bef und: Mittelkräftiger Knochenbau, kein Fettansatz; schwach fassförmiger Thorax, ebenmässig gebaut, Brustbein etwas vorspringend. Die Elastizität verhältnismässig gut erhalten. Atmung von leichtem Stridor begleitet. Meist abdominale Atmung mit Hebung des Brustkorbes, keine Flankenatmung. Lungengrenzen hinten am oberen Rand der 10. Rippe, leidlich verschieblich; ganz geringe Bronchitis; Vitalkapazität 2800 im Mittel. Bauchmuskulatur gut.

\begin{tabular}{lc} 
Neigungswinkel (Freund) & $\begin{array}{c}\text { Sagittaler Durchmesser in der } \\
\text { Neigungsebene }\end{array}$ \\
bei gewönnlicher Atmung $32^{\prime \prime}$ & $13^{3}+\mathrm{cm}$ \\
bei tiefster Inspiration $25^{\circ}$ & $\mathrm{cm}$ \\
\hline
\end{tabular}

Sagittaler horizontaler Durchmesser bei gewöhnlicher Atmung

bei tiefster Inspiration

Oberer Sternalrand $14^{3}+\mathrm{cm}$

Mitte Sternum $22 \mathrm{~cm}$

$15^{1}+\mathrm{cm}$

Processus ensiformis $22^{1}, \mathrm{~cm}$

$22^{1} \cdot \mathrm{cm}$

$22^{3} / \mathrm{cm}$

Umfang in Mamillarlinie bei gewöhnlicher Atmung: $94: 941 / 2 \mathrm{~cm}$ bei tiefster Exspiration und Inspiration: $\quad 93^{1} / 2: 961 / 2 \mathrm{~cm}$ Unfang des Bauches in Nabelhöhe bei gewöhnlicher Atmung: $89: 891 / 2 \mathrm{~cm}$ bei tiefster Exspiration und Inspiration: $86^{1 / 2}: 89^{1}: 2 \mathrm{~cm}$.

Röntgenbefund: Gute Verschieblichkeit des Zwerchfells. Differenz zwischen tiefster Inspirationsstellung und höchster Exspirationsstellung bei $Z$ werchfellkuppe beträgt auf beiden Seiten $51 / 4 \mathrm{~cm}$ auf dem Diagramm. 
Patient leistet durch 15 maliges Ersteigen einer ziemlich steilen Leiter yon $11 / 2 \mathrm{~m}$ Höhe ein gewisses Quantum Arbeit. Bei der Vergleichung dieses Diagramms mit dem vorigen findet sich eine Differenz, indem bei rer tiefsten Inspirationsstellung das Zwerchfell gut tiefer getreten, bei der höchsten Exspirationsstellung dagegen beiderseits am ungefähr $1-1,5 \mathrm{~cm}$ zurückgeblieben ist. Eine zweite Arbeit leistet er dadurch, dass er mit fixierten Füssen ohne Zuhilfenahme der Arme seinen Oberköper aufrichtet und wieder lingsam sinken lässt so lange, bis eine gewisse Ermildung und Anstrengung der Atemtatigkeit eintrat. Am Diagramm konnte vergloichsweise festgestellt werden, dass hierbej durchaus kein Tiefertreten des Zwerchfells eintrat, sondern eine Höherstellung bei tiefster Einatmung, ein Zeichen, dass kein sekundïres Emphysem eintrat. Das Röntgenbild der vorderen Brustwand ergab keinen deutlichen Befund.

5. O. J., 58 Jahre, Grässe $15 \overline{c m}$. Emphysem.

Anamnese: Vater und Nutter an Altersschwäche gestorben. In der Jugend gesund, aber schwächlich, hat deshalb nicht gedient. Sein Teiden kesteht seiner Angabe nach seit 9 Jahren, wo er Lungen- und Rippenfellentzündung iiberstanden habe. Seither Atembeschwerden, Husten, beim Bergsteigen Dyspnoe.

Bef und: Schwächlicher Knochenbau, kein Fett. Brust stark gewölbt infolge starken Hervorspringens der Rippenknorpelansätze besonders oben am Brustbein, das deshalb wie eingesunken erscheint. Die Elastizitiat ist leidlich erhalten, besonders im unteren Abschnitt des Thorax. Atmung etwas beschleunigt, sie hebt dabei den Brustkorb nach oben, erweitert ilın aber auch. Die Lungengrenzen stehen hinten am oberen Rand der 10. Rippe, sind perkutorisch ungefähr $11 / 2 \mathrm{~cm}$ verschieblich nach unten. Kosto-abdominaler A temtypus. Bronchitis. Vitalkapazitit fast konstant $2400 \mathrm{kcm}$. Bauchmuskulatur gut.

\begin{tabular}{|c|c|}
\hline Neigungswinkel (Freund) & $\begin{array}{c}\text { Sagittaler Durchmesser in der } \\
\text { Neigungsebene }\end{array}$ \\
\hline bei gewöhnlicher Atmung $30^{\circ}$ & $12: 2 \mathrm{~cm}$ \\
\hline bei tiefster Inspiration $26^{\circ}$ & $131 / z \mathrm{~cm}$ \\
\hline
\end{tabular}

Sagittaler Lorizontaler Durchmesser bei gow öbulicher Atmung

bei tiefster Inspiration

Oberer Sternalrand $15 \mathrm{~cm}$

Mitte Sternum $20 \mathrm{~cm}$

Processus ensiformis $18 \mathrm{~cm}$

$16 \mathrm{~cm}$

$21 \div \mathrm{cm}$

$19^{3 /} \mathrm{fcm}$

Umfang in Mamillarlinis bei gewöhnlicher Atmung

bei tiefster Lxspiration und Inspiration

$81: 82 \div: \mathrm{cm}$

$80,5: 85 \quad \mathrm{en}$

Umfang des Bauches in Nabelhöhe bei gewöhnlicher Atmung $78: 78,5 \mathrm{~cm}$

bei tiefster Exspiration und Inspiration

$77,5: 78,5 \mathrm{~cm}$.

Rontgonbefund: Das Zwerchfell macht gute In- und Exspirations-Bewegungen. Der höchste Stand bei der Exspiration ist von der tiefsten Inspirationsstellung des Zwerchfells links $4^{3}, \mathrm{~cm}$, rechts $4 \mathrm{~cm}$ entfernt. Dis Rippenknorpel 1 und 2 erscheinen auf der Platte stark verknöchert. 


\section{C. P. $64 \mathrm{~J}$ ahre. Grösse $165 \mathrm{~cm}$. Emphysem.}

Die Anamnese thber Familie ergibt nichts für seine Erkrankung Wesentliches. Er erinnert sich keiner besonderen Erkrankung in der Jugendzeit, hat gedient und ist dann später als Ackerknecht tätig gewesen. Er habe immer an Husten und Auswurf gelitten, schon in seiner Jugend. Seit zwei Jahren Atembeschwerden, bemerkbar besonders beim Steigen und sonstigen Anstrengungen. Wurde am 8. Oktober 1907 in Marburg nach Freund operiert, 1 -4 Rippenknorpelstïcke lechts reseziert, seitdem Nachlassen der Beschwerden.

Befund: Mittelkräftig gebanter Mann, kein Fettansatz. Thorax ist durch die vorgenommene Operation asymmetrisch geworden, linke Seite hervortretend, rechte zu ückgesunken. Hier zieht sich eine Narbe, beginnend $3 \mathrm{~cm}$ unterhalb des Schlüsselheines, $2 \mathrm{~cm}$ seitlich rom oberen Sternalrand und dann dem Sternalrand halbkreisförmig folgend innen an der Namillarlinie vorbei bis $3 \mathrm{~cm}$ unter dieselbe. Die Palpation der nicht operierten linken Seite ergibt ganz starre Thoraxwand, der Thorax wird bei der Einatmung im ganzen in die Höhe geboben, die rechte Seite erscheint, wie schon e.wähnt, eingesunken, macht $z$ war wenig Exkursionen beim Atmen, aber mehr als links. Die Elastizität des Thorax ist aufgehoben; links wie rechts. Es scheint, als wenn auf der operierten Seite die zweite Rippe an der Inzisionstelle eine gewisse Beweglichkeit nach der exspiratorischen Seite $z u$ hätte, bei den anderen lässt sich dies nucht nachweisen. Atmung beschleunigt, von bronchialen Geräuschen begleitet. Die Lungengrenzen sind auf der rechten operierten Seite schlecht verschieblich, die Lungengrenze steht hier am oberen Rand der 11. Rippe. Auf der linken Seite steht die Lungengrenze am oberen Rand der 10. Rippe; bessere Verschieblichkeit. Bauchmuskulatur stark. Vitalkapazitiit $2200 \mathrm{cem}$.

Neigungswinkel (Freund)

bei gewöhnlicher Atmung $35^{\circ}$

bei tiefster Inspiration $30^{\circ}$
Sagittaler Durchmesser in der Neigungsebene
Sagittaler horizontaler Durchmesser bei gewöhnlicher Atmung

Oberer Sternalrand $16,5 \mathrm{~cm}$

Mitte Sternum $28 \mathrm{~cm}$

Processus ensiformis $22 \mathrm{~cm}$
$12 \mathrm{~cm}$

$12,1 \mathrm{~cm}$

Umfang in Mamillarlinie bei gewöhnlicher Atmung:

bei tiefster Inspiration

bei tiefster Exspiration und Inspiration:

Umfang des Bruches in Nabelböhe bei gewöhnlicher Atmung: bei tiefster Exspiration und Inspiration:

$16 \%_{4} \mathrm{~cm}$
$24,5 \mathrm{~cm}$
$22 \% \mathrm{~cm}$

$89: 90^{1}, 2 \mathrm{~cm}$

$89: 91 ; \div \mathrm{cm}$

$86: 86^{1 / 2} \mathrm{~cm}$

$86: 87^{\prime}: 2 \mathrm{~cm}$.

Röntgenbefund: Auf dem Schirm sieht man die rechte Zwerchfellkuppe stark abgeflacht, das Zwerchfell macht auf dieser Seite äusserst geringe Exkursionen, selbst bei tiefster Ein- und Ausatmung. Auf der linken Seite ist die Beweglichkeit grösser, aber auch deutlich herabgesetzt, die Komplementäräume öffnen sich schlecht. Auf der Röntgenplatte sieht man die 1. und 2. Rippe links 
stark verknöchert. Auf der operierten Seite ist der sekundären Bindegewebsund Knochenwucherung halber nichts zu erkennen.

7. K. G., 46 Jahre, Grösse $163 \mathrm{~cm}$. Emphysem.

An amnese über Eltern bietet nichts Bemerkenswertes. Als Kind nie krauk gewesen, auch spater nicht. Als Soldat Brustumfang $88: 96 \mathrm{~cm}$. Vor 6 Jahren habe seine Erkrankung angefangen mit Husten, Beschwerlichkeit beim Atmen, Beschwerden, die sich allmählich zur Atemnot bei jeder Anstrengung geäussert hätten. Gibt der Einatmung vielen Staubes Schuld an seinem Leiden.

Befund: Mittelmässig kräftig gebauter Mann, kein Fettansatz. Brustkasten erscheint mässig erweitert, etwas nach oben gezogen. Die Rippenknorpelansätze sind an beiden Seiten des Brustbeines besonders oben stark hervortretend. Die Elastizität ist gering, besonders in den oberen Abschnitten. Bei tiefem Einatmen wird der ganze Brustkorb nur gehoben, nicht in sich gedehnt, starke Bronchitis. Die Lungengrenzen binten beiderseits am unteren Rande 10. Rippe, sehr schlecht verschieblich, rechts etwas besser wie links. Vitalkapazität im Mittel $2000 \mathrm{ccm}$. Bauchmuskulatur gut.

\begin{tabular}{cc} 
Neigungswinkel (Freund) & $\begin{array}{c}\text { Sagittaler Durchmesser in dex } \\
\text { Neigungsebene }\end{array}$ \\
$\begin{array}{l}\text { bei gewöhnlicher Atmung } 28^{0} \\
\text { bei tiefster Inspiration } 24^{0}\end{array}$ & $12^{1} \mathrm{~cm}$ \\
\hline
\end{tabular}

Sagittaler horizontaler Durclimesser

bei tiefster inspiration

Oberer Sternalrand $15^{1 / 2} \mathrm{~cm}$

Mitte Sternum $22 \mathrm{~cm}$

Processus ensiformis $21 \mathrm{~cm}$
16 I., $\mathrm{cm}$

$231 / 4 \mathrm{~cm}$

$23 \mathrm{~cm}$

Umfang in Mamillarlinie bei gewöhnlicher Atmung: $\quad 86 \quad: 86^{3} / \mathrm{cm}$

bei tiefster Exspiration und Inspiration: $\quad 86: 91 \mathrm{~cm}$

Umfang des Banches in Nabelhöhe bei gewöhnlicher Atmung: $74^{3} / 1: 75 \mathrm{~cm}$

bei tiefster Exspiration und Inspiration: $\quad 75: 74 \mathrm{~cm}$.

Röntgenbefund: Das Zwerchfell zeigt auf beiden Seiten schlechte Verschieblichkeit, rechts etwas mehr wie links, entsprechend dem perkutorischen Befund. Die exspiratorische $Z$ werchfellbewegung geschieht ruckweise. K. G. steigt ziemlich schnell eine $2 \mathrm{~m}$ hohe Leiter siebenmal hinauf und hinab, die $\mathrm{Wwerchfell}$ schattengrenze steht nach dieser Anstrengung in Exspirationsstellung am $2_{1 / 2}^{1}-3 \mathrm{~cm}$ tiefer als vorher. Nach 5 Minuten steht das Zwerchfell noch um $2 \mathrm{~cm}$ tiefer, hat aber nach einer Stunde den alten Stand erreicht. Es hatte sich also hier eine sekundäre Lungenblähung entwickelt.

8. G. U., 40 Jahre, Grösse $158 \mathrm{~cm}$.

Anamnese: Vater und Mutter sind an Asthma gestorben. Geschwister gesund. Als Kind und in der Jugend schwächlich, hat deshalb nicht gedient. 


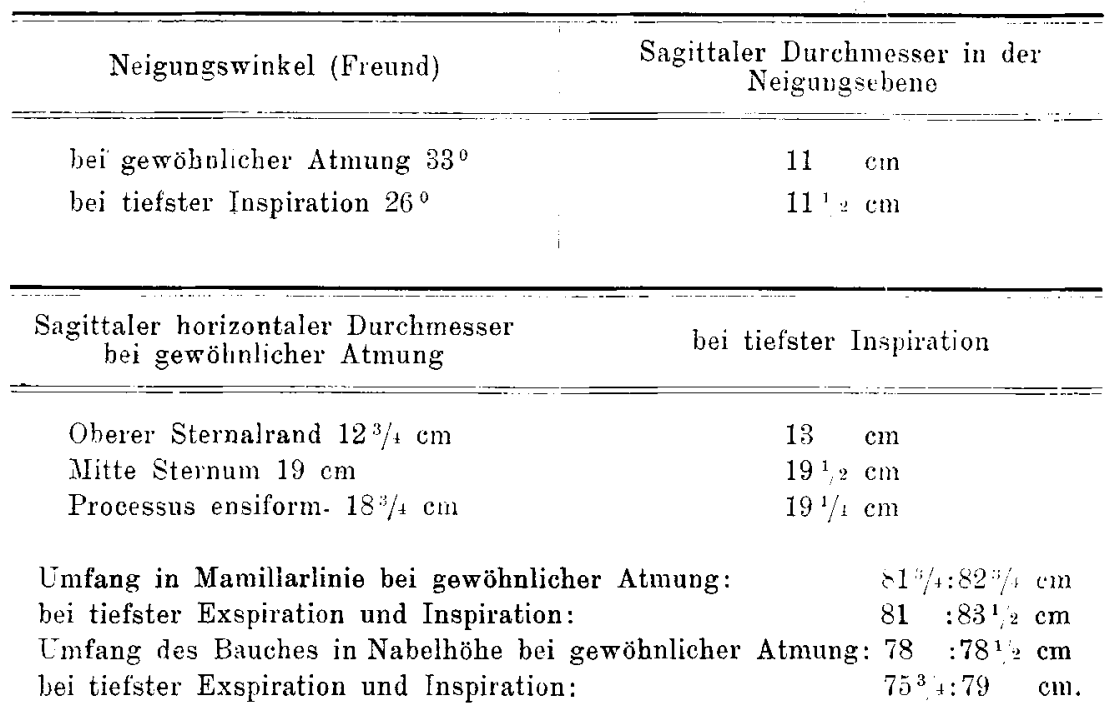

Röntgenbefund: Zwerchfell links mässig, rechts noch schlechter verschieblich, bei tiefster Inspiration und Exspiration rechts $2,2 \mathrm{~cm}$, links $2,7 \mathrm{~cm}$.

10. G. H., 58 Jahre, Grösse $168 \mathrm{~cm}$. Emphysem.

A namnese: Vater und Mutter an Altersschwäche gestorben. Geschwister gesund. Selbst immer gesund. Ist Dienstmann, trägt immer schwere Lasten. Seit etwa 10-12 Jahren Asthma, Husten und Auswurf.

Befund: Kräftige Figur, mässiger Fettansatz, Brustkorb fassförmig, wenig Elastizität erhalten. Atmung abdomial, bei tiefster Atmung wird der Brustkorb mit Anspannung der Halsmuskeln gehoben, schlecht erweitert. Lungengrenzen hinten an 11. Rippe, wenig verschieblich. Bauchmuskulatur gut.

\begin{tabular}{cc} 
Neigungswinkel (Freund) & $\begin{array}{c}\text { Sagittaler Durchmesser in der } \\
\text { Neigungsebene }\end{array}$ \\
\hline
\end{tabular}

bei gewöhnlicber Atmung $36^{\circ} \quad 13 \mathrm{~cm}$

bei tiefster Inspiration $34^{\circ} \quad 13 \mathrm{~cm}$ Sagittaler horizontaler Durchmesser
bei gewöhnlicher Atmung

Oberer Sternalrand $15 \mathrm{~cm} \quad 15 \mathrm{~cm}$

Mitte Sternum $19^{1}{ }_{2} \mathrm{~cm} \quad 20 \mathrm{~cm}$

Processus ensiform. $17{ }^{1} ; 2 \mathrm{~cm} \quad 18 \mathrm{~cm}$

Umfang in Mamillarlinie bei gewöhnlicher Atmung: $\quad 88: 881 / 2 \mathrm{~cm}$

bei tiefster Exspiration und Inspiration: $88: 891 / 4 \mathrm{~cm}$ 
Umfang des Bauches in Nabelhöhe bei gewöhnlicher Atmung: $85: 85^{1}{ }^{1} 2 \mathrm{~cm}$ bei tiefster Exspiration und Inspiration: $84^{1,2}, 85^{1}, 2 \mathrm{~cm}$.

Röntgen befund: Zwerchfell schlecht verschieblich, links 1,4 , rechts $1,0 \mathrm{~cm}$.

11. V. B., 71 Jahre, Grösse $165 \mathrm{~cm}$. Emphysem.

Die Anamnese bietet nichts wesentliches, selbst in der Jugend gesund, aber: schwäehlich. Als Schneider viel zusammengekauert gesessen, seit einigen Jahren etwas Husten, Katarrh, dann Atembeschwerden beim Steigen.

Bef und: Sehr schwächliche abgemagerte Person. Brustkorb stark gewölbt, ist noch nachgiebig. Die Ansatzpunkte der Rippenknorpel am oberen Brustbeinteil stark hervortretend. Abdominale Atmung. Bei tiefstem Atmen beteiligt sich ganz leidlich der Brustkorb. Lungengrenzen hinten unterer Rand 10. Rippe leidlich verschieblich. Bronchialkatarrh. Bauchmuskulatur gut.

\begin{tabular}{|c|c|}
\hline Neigungswinkel (Freund) & $\begin{array}{l}\text { Sagittaler Durchmesser in der } \\
\text { Neigungsebene }\end{array}$ \\
\hline bei gewöhnlicher Atmung $26^{\circ}$ & $12 \mathrm{~cm}$ \\
\hline bei tiefster Inspiration $24^{\circ}$ & $12^{1 / 2} \mathrm{~cm}$ \\
\hline $\begin{array}{c}\text { Sagittaler horizontaler Durchmesser } \\
\text { bei gewöhnlicher Atmung }\end{array}$ & bei tiefster Inspiration \\
\hline Oberer Sternalrand $13,2 \mathrm{~cm}$ & $13^{1} \mathrm{~cm}$ \\
\hline Mitte Sternum $18^{1}, \mathrm{~cm}$ & $19^{1}+\mathrm{cm}$ \\
\hline Processus ensiform. $17^{3}+\mathrm{cm}$ & $18^{1}, 2 \mathrm{~cm}$ \\
\hline
\end{tabular}

Umfang in Mamillarlinie bei gewöhnlicher Atmung:

$77,4: 78,3 \mathrm{~cm}$

bei tiefster Exspiration und Inspiration:

$76,9: 80,4 \mathrm{~cm}$

Umfang des Bauches in Nabelhöhe bei gewöhnlicher Atmung: $75,3: 75,9 \mathrm{~cm}$

bei tiefster Exspiration und Inspiration: $74^{1}, 2: 75,9 \mathrm{~cm}$.

Röntgenbefund: Zwerchfell auf dem Diagramm rechts $3 \mathrm{~cm}, \operatorname{links} 4 \mathrm{~cm}$ verschieblich bei Atemvertiefung, also gute Zwerchfelltätigkeit.

12. J. Z., 64 Jahre. Emphysem.

A n a mese: Vater ist an Lungenschwindsucht gestorben, Mutter an Altersschwäche. Er selbst war in der Jugend gesund, hat gedient, hatte als Maurer schwere Lasten zu tragen. Hat dann die Feldzüge 1866 und 1870 mitgemacht. Seitdem hatte er sein Leiden, Husten, A temnot beim Gehen und Steigen.

Befund: Sehr schwächliche, abgemagerte Gestalt, Brustkorb schwach fassfürmig, Brustbein hervorgewölbt. Die ersten beiden Rippenknorpelansätze am Brustbein sind stark prominent. Die Elastizität ist herabgesetzt, nicht aufgehoben. Bei der Atmung (abdominaler Typus) beteiligt sich der Brustkorb durch Hebung und wird in den oberen Partien aufgeblasen. Die Halsmuskulatur wird beim Atmen stark gespannt. Die Lungengrenzen stehen hinten am oberen Rand der 11. Rippe beiderseits, geringe Verschieblichkeit nach der in- und exspiratorischen Seite hin. Bronchitis, Bauchmuskulatur gut. 


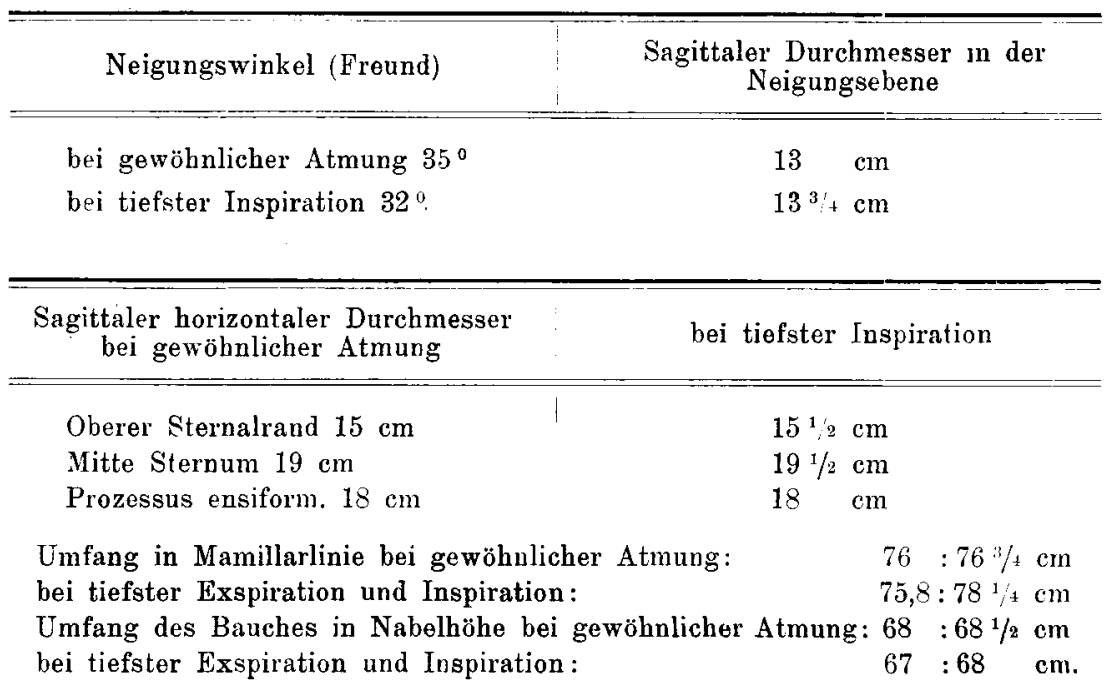

Rön tgen befund: Zwerchfell bei ruhiger Atmung wenig verschieblich, links und rechts je $1,0 \mathrm{~cm}$.

\section{Literaturverzeichnis.}

1. Chr. Bohr, Die funktionellen Änderungen in der Mittellage und Vitalkapazität der Lungen. Deutsch. Arch. für klin. Medizin. Bd. 88. 1907.

2. Derselbe, Zur Theorie der Entstehung des Lungenemphysems. Zentralbl. für die ges. Fhysiologie und Pathologie des Stoffwechsels. $1908 \mathrm{Nr} .7$.

3. L. Brauer. Der Druck zwischen den Pleurablättern. Beiträge zur pathol. Anatomie und zur allgem. Pathologie. 7. Supplement.

4. Bruns, Nachweis, Entstehung und Bedeutung der temporären Lungenblähung. Zeitschr. für experimentelle Pathologie und Therapie 1909.

5. Cohn, Zur Operation des Lungenemphysems (W. A. Freund). Deutsch. med. Wochenschr. 1908. Nr. 10.

6. Freund, Beiträge zur Histologie der Rippenknorpel im normalen und pathologischen Zustand. Breslau 1838.

7. Derselbe, Der Zusammenlang gewisser Lungenkrankheiten mit primären Rippenknorpelanomalien. Erlangen 1859.

8. Derselbe, Thoraxanomalien als Prädisposition zur Lungenphthise und Emphysem. Berl. klin. Wochenschr. 1901.

9. Derselbe, Über primäre Thoraxanomalien, speziell über die starre Dilatation des Thorax als Ursache eines Lungenemphysems. Berlin 1906. Verlag S. Kaiser.

10. Delselbe, Zur operativen Behandlung gewisser Lungenkrankheiten, insbesondere auf starrer Thoraxdilatation beruhenden alveolären Emphysems 
(mit einem Operationsfalle). Zeitschr. für experimentelle Pathologie und Therapie 1906. Bd. 3. S. 479.

11. Freund, Beiträge zur Behandlung der tuberkulösen Lungenspitzenphthise und des alveolären Emphysems durch operative Mobilisation des in der ab. Apertur stenosierten und des starr dilatierten Thorax. Münch, med. Wochenschrift 1907. Nr. 48.

12. Derselbe, Die Beziehung der Heilungsvorgänge gewisser Formen der Lungenphthise zur Gelenkbildung am ersten Rippenringe. Therapeutische Nonatshefte 1902. Juni.

13. Freund und Mendelsohn, Der Zusammenhang des Infantilismus des Thorax und des Beckens. Stuttgart. Verlag von F. Enke. 1908.

14. Grawitz, Über Lungenemphysem. Deutsche med. Wochensehr, 1892. 18. 10.

15. Hart, Karl, Zur Frage der chirurgischen Behandlung der beginnenden tuberkulösen Lungenspitzenphthise. Münch. med. Wochenschr. 1907. Nr. 44.

16. Hofbauer, Zur Frage nach der Ęntstehung von Lungenblähung. Deutsche med. Wochenschr. 1908. Nr. 51.

17. Derselbe, Wann ist bei chronischem Lungenleiden (Emphysem, Tuberkulose) operative Behandlung indiziert? Mitteil. aus dem Grenzgeb. der Medjz. und Chir. 1908. Bd. 18. H. 5.

18. Derselbe, Mechanik der Respirationsstörungen bei pleuralen Erkrankungen und die Dyspnoe bei der exsudativen Pleuritis. Zentralbl. f. inn. Med. 1906.

19. Derselbe, Mechanik des Lungenemphysems. Wiener mediz. Wochenschr. 1907. Nr. 45.

20. Derselbe, Physikalische Therapie des Lungenemphysems. Wiener mediz. Wochensehr. 1908. Nr. 6.

21. Dersejbe, Mechanik der Respirationsstörung bei pleuralen Erkrankungen und die Dyspnoe beim Pneumothorax. Zentralbl. f. inn. Med. 1905.

22. Derselbe, Zur operativen Behandlung gewisser Lungenkrankheiten (Emphysem und Tuberkulose). Zeitschr. f. experiment. Path. u. Therap. 1907. S. 198.

23. Hofbauer und Holzknecht, Zur Physiologie und Pathologie der Atmung. Mitteil. a. d. Laborat. f. radiol. Diagnost. u. Ther. im K. K. allgem. Krankenhaus in Wien 1907.

24. Hof fmann, Emphysem und Atelektase. Nothnagel, Spez. Path. u. Ther. 1900. Bd. 14. 1. Abt.

25. H o p pe-Seyler, Die verschiedenen Arten von Lungenerweiterung, besonders des komplementären (vikariierenden) Emphysens. Münch. med. Wochenschr. 1909. Nr. 2. S. 104.

26. Klamperer, Die chirurgische Behandlung gewisser Formen von Lungenemphysem. Freund sche Operation. Lejars Sem. med. 1907. Nr. 45.

27. Klasi, Anatomische Untersuchungen über die Entstehung des vesikulären Lungenemphysems. Virch ow A Arch. 1886. Bd. IV. 2. S. 353.

28. Krehl, Pathologische Physiologie. 1904. 3. Autl. S. 244.

29. Liebermeister, Zur normalen und pathologischen Physiologie der Atmungsorgane. 2 Studien über die Atmungsmechanik bei plötzlich auftretender Larynxstenose. Deutsche med. Wochenschr. Nr. 39.

30. Derselbe, Über Lungenemphysem. Deutsche med. Wochenschr. 1891. 17. 12.

31. Derselbe. Über das Verhältnis zwischen Lungendehnung und Lungenvolumen. Zentralbl. f. Path. u Anat. 1907. S. 644.

32. Luciani, Die Mechanik der Atmung. - Die Physiologie des Menschen. Bd. 1. 1905. S. 331. 
33. Mohr, Zur Pathologie und Therapie des alveolären Emphysems. Berl. kliu. Wochenschr. 1907. Nr. 27.

34. Mueller, Zur operativen Behandlung des Lungenemphysems. Zentralbl. f. d. Grenzgeb. d. Med. u. Chir. 1908. Bd. 11. Nr. 9.

35. Nager, Über primäre Geschwälste der Trachea. Med. Klin. 1909. Nr, 1. S. 23.

36. Pässler und Seidel, Beitrag zur Pathologie und Therapie des alveolären Lungenemphysems. Münch. med. Wochenschr. 1907. Nr. 38.

37. Schenk, Atembewegungen. Tigerstedt, Handbuch d. phys. Methodik.

38. Dersel be, Innervation der Atmung. Asher-Spiro, Ergebnisse der Physiologie. 7. Jahrg.

39. Schlippe; Physikalische Untersuchungen bei der Anwendung des Magenschlauches. Deutsch. Arch. f. klin. Med. Bd. 76.

40. Stieda, Über die Behandlung gewisser Fälle von Lungenemphysem. Münch. med. Wochenschr. 1907. Nr. 38.

41. Tendeloo, Die funktionelle Bedeutung des Lungenvolumens in normalen und pathologischen Zuständen. Gesellschaft deutsch. Naturforscher und Ärzte. Verhandlungen 1907. Verlag G. Vogel.

42. Derselbe, Studien über die Ursachen der Lungenkrankheiten. Über die Ursachen des Emphysems. S. 1-187. 1902.

43. Tigerstedt, Der kleine Kreislauf. Ergebnisse der Physiologie. Bd. 2. S. 529 .

44. Volh ard, Verhandlungen der Gesellschaft deutscher Naturforscher und Ärzte. Dresden 1907.

45. Wenkebach, Über pathologische Beziehungen zwischen Atmung und Kreislauf beim Menschen. Sammlung klin. Vorträge. Neue Folge 1907. Verlag Breitkopf und Härtel, Leipzig.

46. Wisske, Über Geschichto und path. Veränderungen des Lungenemphysems. Inaugural-Dissertation 1892. 